\title{
Editorial
}

\section{Strengthening nurses' resilience and reducing burnout during the COVID-19 pandemic}

\author{
Mirko Prosen \\ University of Primorska, Faculty of Health Sciences, Izola, Slovenia
}

The COVID-19 pandemic presents a significant health burden to healthcare systems worldwide. At the time of writing this editorial, the pandemic continues to escalate all across Europe, posing additional concerns to already understaffed health systems. As the problem is multidimensional in nature, the shortage of nursing staff in particular has been a global challenge for at least two decades - and will continue to be so for some time to come. To date, there really has been no effective strategy to tackle this issue on a global scale, and the COVID-19 pandemic has further exacerbated the problem of understaffing in the worst possible way. There is ample evidence that adequate nurse staffing is associated with positive patient outcomes, including lower mortality, fewer complications, higher patient satisfaction, shorter hospital stays and fewer readmissions, as well as better outcomes for nurses, such as less burnout (Lasater et al., 2020). The latter has now become a major concern, as nurses (as well as other healthcare workers), most of whom provide holistic care on the frontline in the battle against the COVID-19 pandemic, are increasingly overburdened, exhausted and under extended workplace stress. The results can be devastating. Ross (2020), for example, points out that although the link between suicide and burnout among nurses has not been sufficiently investigated, we may assume that nurses are at a higher risk of suicide. I do not wish for this editorial to sound too pessimistic, but the fact is that this topic needs to be openly discussed within the profession, within healthcare teams and among those whose decisions affect the work of nurses on a daily basis.

Burnout is the result of prolonged workplace stress that is not managed effectively. It is a stress response similar to post-traumatic stress syndrome, which affects not only one's personal wellbeing, but also patient wellbeing and outcomes as it affects the delivery of compassionate and quality healthcare (Hofmeyer et al., 2020; Ross, 2020). It is therefore important to raise the question of the impact the COVID-19 pandemic and the associated workload might have on nurse burnout. Beyond any doubt, natural disasters such as pandemics present a significant psychological burden. As Manzano-García and Ayala-Calvo (2020) eloquently explain, pandemics exacerbate the stress nurses feel when confronted with suffering, pain and death, as well as with ethical dilemmas arising from these situations. Furthermore, material and human resources are often scarce, leaving nurses to work in an unstable environment and under a heavy workload, with lack of control over patient flow, poor management and lack of planning. An additional emotional burden for nurses is the fear of bringing the infection into their home and exposing their family members to COVID-19 (Bagnasco et al., 2020). In view of all this, it is safe to assume that, with the prolongation of the pandemic, the wellbeing of nurses will deteriorate at a much faster rate, which in turn will affect not only nurses but also the already vulnerable health system.

Therefore, appropriate strategies need to be considered which will support nurses' mental, psychological and emotional health and enhance their personal resilience, help them effectively endure prolonged stress and recover from stressful situations much more quickly. Among these, there are three comprehensive resilience strategies offered by the Mayo Clinic which deserve our attention. These strategies focus on (1) modelling resilience strategies, (2) establishing strong peer support and stress management programmes, and (3) promoting organisational resilience (Chesak et al., 2020). Resilient leadership relies on managers who empower and lend credibility during times when courage, vision and values of camaraderie must be at the forefront of the fight against the COVID-19 pandemic. Social support from colleagues and managers (not to mention friends and family) presents a valuable and effective coping mechanism, which must be constantly encouraged and supported within individual healthcare teams and organisations. This also includes the provision of psychological first aid and counselling for all healthcare professionals. Organisational resilience is characterised by embracing imbalance and mastering agility, adaptability and leadership at all levels. Organisations which fail to respond effectively to this crisis and disregard lessons learned during the crisis (i.e., the initial wave of COVID-19) and lose focus, will not be able to function effectively and reduce the impact of various workplace stressors (Chesak et al., 2020; Labrague and De los Santos, 2020).

In the midst of all this, the role of nurse managers must also be considered. Not only from the perspective of their

\footnotetext{
* Corresponding author: Mirko Prosen, University of Primorska, Faculty of Health Sciences, Izola, Slovenia; e-mail: mirko.prosen@fvz.upr.si http://doi.org/10.32725/kont.2021.001 
responsibilities, but also from the perspective of their own resilience to workplace stressors, which are indirectly associated with fulfilling their responsibilities. When we discuss the strategies to address nurse burnout, the role of nurse managers is perhaps something which needs to be addressed first. Namely, as pointed out by Hofmeyer et al. (2020): "We are told to put on our own oxygen 'mask' before helping others during a flight emergency."

During the year in which we celebrate the International Year of the Nurse and the Midwife, which also coincides with the 200th anniversary of the birth of the founder of modern nursing Florence Nightingale, the nursing profession is undoubtedly confronted with its greatest challenges in human history. As the largest group of healthcare professionals within the health system, nurses are at the centre of the fight against the COVID-19 pandemic. Working day and night to provide patients with holistic care, oftentimes in inhumane conditions and without sufficient and adequate personal protective equipment, they require high levels of personal resilience in order to effectively prevent burnout. However, every nurse, like every human being, can only resist workplace-related stress up to a point. Let this editorial be a call to action for every nurse and nursing manager to not neglect their own personal physical and psychosocial wellbeing, and at the same time for all health institution managers and other key stakeholders within the health system to not overlook their role in securing a collectively supportive work environment. The lesson must finally be learned. We simply cannot wait for the next natural disaster to show us that our profession is fundamental and valuable to society, and that securing a sufficient number of nurses to meet the healthcare needs of all people is a sensible investment in our future.

\section{References}

1. Bagnasco A, Zanini M, Hayter M, Catania G, Sasso L (2020). COVID 19-A message from Italy to the global nursing community. J Adv Nurs 76(9): 2212-2214. DOI: 10.1111/ jan.14407.

2. Chesak SS, Perlman AI, Gill PR, Bhagra A (2020). Strategies for Resiliency of Medical Staff During COVID-19. Mayo Clin Proc 95(9): S56-S59. DOI: 10.1016/j.mayocp.2020.07.002.

3. Hofmeyer A, Taylor R, Kennedy K (2020). Fostering compassion and reducing burnout: How can health system leaders respond in the Covid-19 pandemic and beyond? Nurse Educ Today 94: 104502. DOI: 10.1016/j.nedt.2020.104502.

4. Labrague LJ, De los Santos JAA (2020). COVID-19 anxiety among front-line nurses: Predictive role of organisational support, personal resilience and social support. J Nurs Manag 28(7): 1653-1661. DOI: 10.1111/jonm.13121.

5. Lasater KB, Aiken LH, Sloane DM, French R, Martin B, Reneau K, et al. (2020). Chronic hospital nurse understaffing meets COVID-19: an observational study. BMJ Qual Saf bmjqs-2020-011512. DOI: 10.1136/bmjqs-2020-011512.

6. Manzano-García G, Ayala-Calvo JC (2020). The threat of COVID-19 and its influence on nursing staff burnout. J Adv Nurs. DOI: 10.1111/jan.14642.

7. Ross J (2020). The Exacerbation of Burnout During COVID-19: A Major Concern for Nurse Safety. J Perianesth Nurs 35(4): 439-440. DOI: 10.1016/j.jopan.2020.04.001. 